\title{
THE CLOSED-FORM EXPRESSIONS FOR THE NEUTRON AND X-RAY REFLECTION AND TRANSMISSION COEFFICIENTS OF A ONE DIMENSIONAL PROFILE
}

\author{
XIAO-L!N ZHOU ${ }^{1},+$, GIAN P. FELCHER ${ }^{2}$, AND SOW-HSIN CHEN ${ }^{+}$ \\ ${ }^{1}$ IPNS Division, and ${ }^{2}$ Materials Science Division, \\ Argonne National Laboratory, \\ Argonne, IL 60439 \\ +Department of Nuclear Engineering, \\ Rm 24-209, \\ Massachusetts Institute of Technology, \\ Cambridge, MA 02139

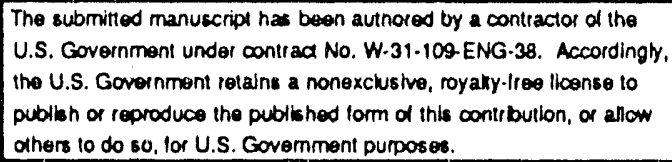 \\ ahers to do so, lor U.S. Government purposes.<smiles>[Y11]1CCCC1</smiles> \\ DEC 0 \& 1990 \\ CONE $-9008161--2$ \\ DE91 004453
}

November, 1990

Submitted to Physica Review B

\section{DISCLAIMER}

\begin{abstract}
This report was prepared as an account of work sponsored by an agency of the United States Government. Neither the United States Government nor any agency thereof, nor any of their employees, makes any warranty, express or implied, or assumes any legal liability or responsibility for the accuracy, completeness, or usefulness of any information, apparatus, product, or process disclosed, or represents that its use would not infringe privately owned rights. Reference herein to any specific commercial product, process, or service by trade name, trademark, manufacturer, or otherwise does not necessarily constitute or imply its endorsement, recommendation, or favoring by the United States Government or any agency thereof. The views and opinions of authors expressed herein do not necessarily state or reflect those of the United States Governmemt or any agency thereof.
\end{abstract}

Proceedings from the Workshop on Mlithods of Analysis and Interpretation of Neutron Reflectometry Data , August 23-25, 1990, Argonne National Laboratory, Argonne, IL

This work is supported by the U.S. Department of Energy, Basic Energy Sciences, 


\title{
THE CLOSED-FORM EXPRESSIONS FOR THE NEUTRON AND X-RAY REFLECTION AND TRANSMISSION COEFFICIENTS OF A ONE DIMENSIONAL PROFUE
}

\author{
XIAO-LIN ZHOU $1,+$, GIAN P. FELCHER ${ }^{2}$, AND SOW-HSIN CHEN ${ }^{+}$ \\ ${ }^{1}$ IPNS Division, and 2Materials Science Division, Argonne National Laboratory, \\ Argonne, IL 60439 \\ +Department of Nuclear Engineering, Rm 24-209, Massachusetts Institute \\ of Technology, Cambridge, MA 02139
}

\section{ABSTRACT}

As the first part of an effort to systematically study the inversion problem in $\mathrm{x}$-ray and neutron reflectivity experiments, the closed-form expressions are derived for the reflection and transmission coefficients as functionals of the sample profile. The assumption used is that the reflection is mainly due to the first- and the second-order derivatives of the profile and thus the third- and higher-order derivatives are negligible. One of the two major characteristics of the formulas is that the reflection and transmission coefficients are explicitly expressed in terms of the profile; the other is that the formulas are valid over the entire range of momentum transfer $Q$. This procedure enables the straight-forward calculation of the real space profile using the reflectivity data as the computer input, with an accuracy that still remains to be evaluated through both analytical and numerical analyses with the aid of model profiles.

Keywords neutron, $\mathrm{x}$-ray, reflection, reflectivity, inversion 


\section{INTRODUCTION}

The reflection of $\mathrm{x}$-rays and neutrons has become a powerful and versatile technique to probe density profiles $[1,2,3,4]$. However, the processing of the reflectivity data turns out to be difficult and time-consuming as the methods in use are all in essence some sort of fitting routines $[1,5]$ using least-square criteria on a model sample profile. An ill-understood sample with a very complex structure would practically prohibit successful data fitting, limiting the usefulness of the reflection technique.

The inversion method presented here is the straight-forward numerical calculation of the sample profile with the reflectivity data taken as the computer input. At present, rigorous closed-form expressions for the reflectivity and transmissivity are unavailable. Therefore, the purpose of this paper is to provide such closed-form expressions for smoothly varying potentials.

In the reflection theory, the waves in a one-dimensional profile is wellunderstood to be governed by the famous Helmholtz equation [6,7]. Unfortunately, there is no exact analytic solution to this equation for an arbitrary profile and an approximation must be sought. The criteria for making approximations are expertly exposed by Lekner [7], where the longand the short-wave approximations are obtained with the former being essentially the Born approximation while the latter the WKB highfrequency approximation. Although it is fairly accurate at large Q's, the popular Born approximation, as a single-reflection approximation, can not account for the near-total-reflection behavior. On the other hand, the highfrequency approximation is the ray propagation result. Neither can accurately describe the reflection in the entire range of $Q$ values. Since the inversion method requires the closed-form expression for the reflection and transmission coefficients which applies to the whole range of $Q$ values, the development of new closed-form formulas are needed.

In the following, the open-form wave functions and the reflection and transmission coefficients are first derived in section 2 from the Helmholtz wave equation with the help of Green's function. Then the wave in the sample is approximately solved for in a closed form in section 3. Thirdly, the closed-form wave function inside the sample is used to derive the closed- 
form expressions for the reflection and transmission coefficients in section 4.

\section{REFLECTION AND TRANSMISSION COEFFICIENTS : OPEN-FORM EXPRESSIONS}

The geometry of the reflection and transmission of a plane wave is illustrated in Fig.1, where region 1 is the free space, region 2 is the sample layer which extends from $\mathbf{z}=-\mathbf{d}$ to $\mathbf{z}=0$, region 3 is the substrate and the $\mathbf{z}$ components of the wave vectors in the three regions are $\mathbf{k}_{\mathbf{o}}, \mathbf{k}$ and $\mathbf{k}_{\mathbf{S}}$, respectively. $\theta$ denotes the incident angle, $\mathbf{d}$ the layer thickness, $\mathbf{r}$ the reflection coefficient, $\mathbf{t}$ the transmission coefficient and $\mathbf{U}(\mathrm{z})$ the wave function. In the following derivation, the subscript ' $o$ ' and ' $s$ ' stand for 'free-space' and 'substrate' while the parameters without subscripts are pertinent to the sample layer.

The one-dimensional Helmholtz equations satisfied by the waves in the three regions are ${ }^{[7]}$

$$
\begin{aligned}
& U^{\prime \prime}(z)+k_{o}^{2} U(z)=0, \text { for } z \leq-\mathrm{d}, \text { in the free space } \\
& U^{\prime \prime}(z)+k_{o}^{2} U(z)=-\left(k^{2}-k_{o}^{2}\right) U(z), \text { for }-\mathrm{d} \leq \mathrm{z} \leq 0, \text { in the sample } \\
& U^{\prime \prime}(z)+k_{s}^{2} U(z)=0, \text { for } 0 \leq \mathrm{z}, \text { in the substrate }
\end{aligned}
$$

where the double prime denotes the second derivative with respect to $\mathrm{z}$. The wave numbers $\mathrm{k}_{0}, \mathrm{k}$ and $\mathrm{k}_{\mathrm{S}}$ have the following expressions for the neutron[8]

$$
\begin{aligned}
& k_{o}^{2}=2 m E_{k} / \hbar^{2}=(2 \pi \sin \theta / \lambda)^{2}=(Q / 2)^{2} \\
& k^{2}=k_{o}^{2}-2 m V / \hbar^{2} \\
& k_{s}^{2}=k_{o}^{2}-2 m V_{s} / \hbar^{2}
\end{aligned}
$$




$$
\begin{aligned}
& V=\left(\check{n}^{2} / 2 m \pi\right) N(z) b \\
& V_{s}=\left(h^{2} / 2 m \pi\right) N_{s} b_{s} \\
& f(z) \equiv k^{2}-k_{o}^{2}=-4 \pi N(z) b
\end{aligned}
$$

where $\mathrm{m}$ is the mass of the neutron, $\lambda$ and $\mathrm{E}_{\mathbf{k}}$ its DeBroglie wavelength and $z$-direction kinetic energy in free space, respectively; $V$ the optical potential for the neutron, which san be expressed in terms of $\mathrm{N}(\mathrm{z})$, the atomic density, and $b$, the srattering length, in the sample. The difference of $\mathbf{k}_{\mathbf{o}} \mathbf{2}$ and $\mathbf{k}^{\mathbf{2}}$ is defined as the profile function $\mathrm{f}(\mathrm{z})$ which contains all the information about the sample and is the very thing we will be after in doing inversion. The wave numbers for the $x$-ray are related to the free-space wavelength $\lambda$, the number density of electrons $\rho(z)$, the classical radius of electron $r_{0}$ and the refractive index $n$ as ${ }^{[9]}$

$$
\begin{aligned}
& k_{o}^{2}=(2 \pi \sin \theta / \lambda)^{2} \\
& k^{2}=k_{o}^{2}-(2 \pi / \lambda)^{2} \cdot\left(n^{2}-1\right) \\
& k_{s}^{2}=k_{o}^{2}-(2 \pi / \lambda)^{2} \cdot\left(n_{s}^{2}-1\right) \\
& n=1-\left(\lambda^{2} / 2 \pi\right) \rho(z) \cdot r_{o} \\
& n_{s}=1-\left(\lambda^{2} / 2 \pi\right) \rho_{s} \cdot r_{o} \\
& f(z) \equiv k^{2}-k_{o}^{2} \approx-4 \pi \rho(z) \cdot r_{o}
\end{aligned}
$$

The profile functions Eq.(2.f) and Eq.(3.f) for the x-ray and the neutron are analogous. For magnetic samples, an extra magnetic term should be added to the potential $\mathrm{V}$ in Eq.(2.d).

With the aid of the half-space Green's function[12], one can obtain the following open-form solution for the wave functions in the three regions indicated in Fig. 1 (cf. Appendix A) 
$U(z)=e^{i k_{o} z}+R \cdot e^{-i k_{o} z}+\frac{i}{2 k_{o}} \int_{-d}^{0} d z^{\prime} \cdot f\left(z^{\prime}\right) \cdot U\left(z^{\prime}\right)\left[e^{i k_{o}\left(z^{\prime}-z\right)}+R \cdot e^{-i k_{o}\left(z^{\prime}+z\right)}\right]$

for $\mathrm{z} \leq-\mathrm{d}$, in the free space

$U(z)=e^{i k_{o} z}+R \cdot e^{-i k_{o} z}+\frac{i}{2 k_{o}} \int_{-d}^{0} d z^{\prime} \cdot f\left(z^{\prime}\right) \cdot U\left(z^{\prime}\right)\left[e^{i k_{o}\left|z^{\prime}-z\right|}+R \cdot e^{-i k_{o}\left(z^{\prime}+z\right)}\right]$

for $-\mathrm{d} \leq \mathrm{z} \leq 0$, in the sample

$U(z)=T \cdot e^{i k_{s} z}+\frac{i}{2 k_{o}} T \cdot \int_{-d}^{0} d z^{\prime} \cdot f\left(z^{\prime}\right) \cdot U\left(z^{\prime}\right) e^{i k_{s} z-i k_{o} z^{\prime}}$

for $0 \leq z$, in the substrate

where $R, T$ are the reflection and transmission coefficients for the bare substrate:

$R=\frac{k_{o}-k_{s}}{k_{o}+k_{s}}$

$T=\frac{2 k_{o}}{k_{o}+k_{s}}$

If Eqs.(4.a), (4.b) and (4.c) are substituted into Eqs. (1.a), (1.b) and (1.c), their correctness can be seen right away. Note that Eq.(4.b) agrees with the perturbation theory by Lekner (pp62-63 in [7]).

According to the definition of the reflection and transmission coefficients $\mathbf{r}$ and $\mathbf{t}$, the wave in the free space region 1 and the substrate region 3 should be

$$
\begin{aligned}
& U(z)=e^{i k_{o} z}+r \cdot e^{-i k_{o} z} \\
& \text { for } z \leq-\mathrm{d}, \text { in the free space } \\
& U(z)=t \cdot e^{i k_{s} z} \\
& \text { for } 0 \leq \mathrm{z} \text {, in the substrate }
\end{aligned}
$$


Equating (5.a) to (4.a) and (5.b) to (4.c), one gets

$$
\begin{aligned}
& r-R=\frac{i}{2 k_{o}} \int_{-d}^{0} d z^{\prime} \cdot f\left(z^{\prime}\right) \cdot U\left(z^{\prime}\right)\left[e^{i k_{o} z^{\prime}}+R \cdot e^{-i k_{o} z^{\prime}}\right] \\
& t-T=\frac{i}{2 k_{o}} T \cdot \int_{-d}^{0} d z^{\prime} \cdot f\left(z^{\prime}\right) \cdot U\left(z^{\prime}\right) e^{-i k_{o} z^{\prime}}
\end{aligned}
$$

Clearly, if $U(z)$ inside the sample layer is known, this can be substituted into Eqs.(6.a) and (6.b) to obtain the closed-form expressions for $\mathbf{r}$ and $\mathbf{t}$. In the next section, an approximate expression for the wave function $U(z)$ inside the sample will be derived.

It is worth noting that the physical meaning of Eqs.(4.a) to (1.c) and (6.a) and (6.b) is that the wave in the sample is equal to the wave that would exist in the absence of the sample (i.e. in the presence of the substrate alone) plus the effect of the sample. The difference between the reflection and transmission coefficients of the sample-substrate combination and those of the substrate alone is completely due to the sample profile $f(z)$, and in Eqs.(6.a) and (6.b), has the form of linear iniegrals. The separation of the substrate contribution from the total effect is both enlightening and practical because the formulae are self-evident in their physical implications and suggest how a closed form solution could be obtained; even more, most samples can be taken as the superposition of an unknown profile on a convenient substrate. In addition, the first-order solution that can easily result from Eq.(4) through the standard perturbation procedure is more accurate than the bare Born approximation since the substrate reflection is accounted for from the beginning.

\section{CLOSED-FORM APPROXIMATE SOLUTION FOR THE WAVE IN THE SAMPLE}

Eq.(4.b) does not have a general closed-form solution for an arbitrary profile $f(z)$ except when $f(z)$ is some simple function ( e.g. linear function, or exponential ) and therefore a closed-form solution can be obtained only in an approximate way. The approximation should be at the same time 
satisfactory in the near-total-reflection region and reduce to the first-order approximation at large $Q$ values and for thin samples. As the intense signal near total reflection comes about as a result of multiple reflections, the perturbation (or single reflection) method is not applicable although it is satisfactory at large $Q$.

We would like then to express the wave function as the sum of the firstorder approximation and the multiple-reflection functions $\mathrm{A}(\mathrm{z})$ and $\mathrm{B}(\mathrm{z})$ and reduce the problem to that of solving the multiple-reflection functions (in this way, it is guaranteed that the final formula reduces to the firstorder approximation). What are the quantities which can be approximated equally for the near-total-reflection small $Q$ values as well as the large $Q$ values? The quantity which does not vary when the $Q$ varies from total reflection to large values is the sample profile $\mathrm{f}(\mathrm{z})$. Is it legitimate and possible to make assumptions about the profile? The answer is usually positive. This is because most of the physical problems consist in determining the shape of continuous profiles, which might be due to diffusion, or partial precipitation, or magnetization, into a frame of rigid boundaries, or fixed "substrates". In these circumstances, the third and higher-order derivatives of the smooth profile can be neglected. One can then greatly simplify the equations and make the equations analytically solvable.

Eq.(4.b) can be put in the following form

$$
\begin{aligned}
& U(z) \equiv a(z) e^{i k_{o} z}+b(z) e^{-i k_{o} z} \\
& a(z)=1+\frac{i}{2 k_{o}} \int_{-d}^{z} d z^{\prime} \cdot f\left(z^{\prime}\right)\left(1+r e^{-i 2 k_{o} z^{\prime}}\right)+A(z) \\
& b(z)=\frac{t}{T} R+\frac{t}{T} \frac{i}{2 k_{o}} \int_{z}^{0} d z^{\prime} \cdot f\left(z^{\prime}\right)\left(e^{i 2 k_{o} z^{\prime}}+R\right)+B(z)
\end{aligned}
$$

In Eqs.(7.b) and (7.c), a(z) and b(z) each contain three parts, namely, the constant part for the substrate reflection, the single integral for the first- 
order reflection and the functions $\mathrm{A}(\mathrm{z})$ and $\mathrm{B}(\mathrm{z})$ for the multiple-reflection functions. Through the approximation discussed above, $A(z)$ and $B(z)$ are obtained as (cf. Appendix B)

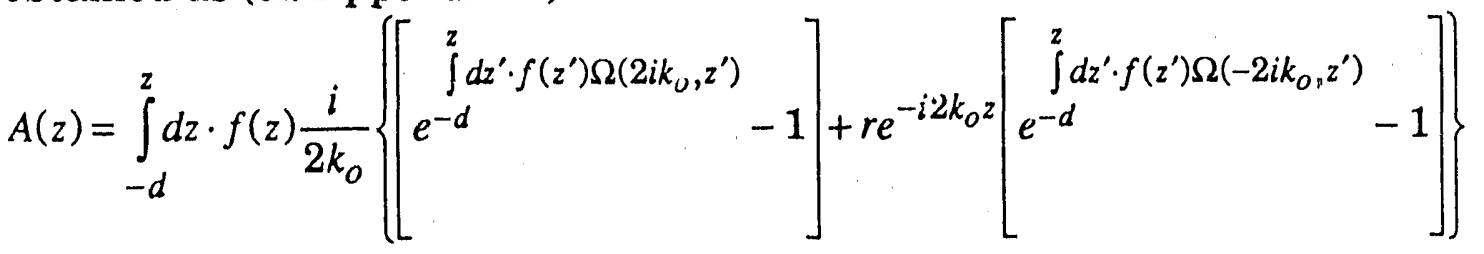

$$
\begin{aligned}
& B(z)=\int_{z}^{0} d z \cdot f(z) \frac{i}{2 k_{o}} \frac{t}{T}\left\{R\left[e^{-\int^{0} d z^{\prime} \cdot f\left(z^{\prime}\right) \Omega\left(-2 i k_{o}, z^{\prime}\right)}-1\right]+e^{i 2 k_{o} z}\left[e^{-\int d z^{\prime} \cdot f\left(z^{\prime}\right) \Omega\left(2 i k_{o}, z^{\prime}\right)}-1\right]\right\}
\end{aligned}
$$

Substituting Eqs.(8.a) and (8.b) into Eqs.(7.b) and (7.c), one has

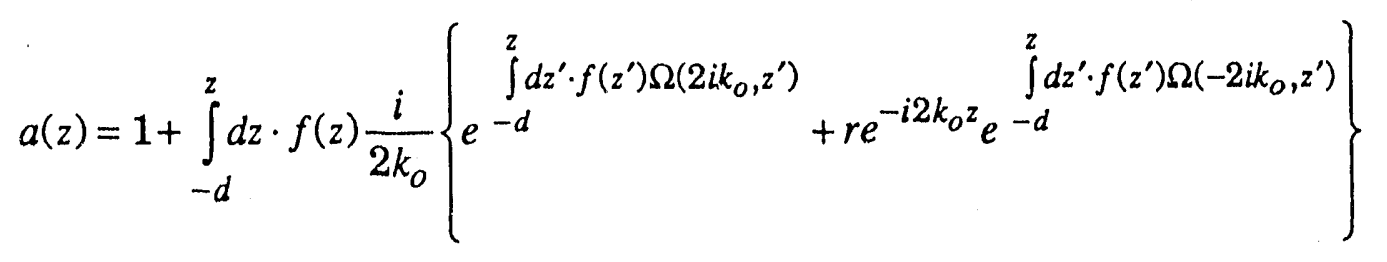

$$
\begin{aligned}
& b(z)=\frac{t}{T} R+\int_{z}^{0} d z \cdot f(z) \frac{i}{2 k_{o}} \frac{t}{T}\left\{\mathrm{R} e^{-\int^{0} d z^{\prime} \cdot f^{\prime}\left(z^{\prime}\right) \Omega\left(-2 i k_{o}, z^{\prime}\right)}+e^{i 2 k_{o} z} e^{-\int^{0} d z^{\prime} \cdot f\left(z^{\prime}\right) \Omega\left(2 i k_{o}, z^{\prime}\right)}\right\}
\end{aligned}
$$

Equations (9.a), (9.b) and Eq.(7.a) give the closed-form solution for the wave function in the sample.

\section{CLOSED-FORM EXPRESSIONS FOR THE REFLECTION AND TRANSMISSION COEFFICIENTS}

Substituting the wave function Eq.(7.a) plus Eqs.(9.a) and (9.b) into Eqs.(6.a) and (6.b), one has a set of simultaneous equations for $r$ and $t$ 
$a_{11} \frac{r}{R}+a_{12} \frac{t}{T}=c_{1}$

$a_{21} \frac{r}{R}+a_{22} \frac{t}{T}=c_{2}$

the solution of which is

$r=M_{r}(Q, f) \cdot R$

$t=M_{t}(Q, f) \cdot T$

with

$M_{r}(Q, f) \equiv \frac{r}{R}=\frac{a_{22} \cdot c_{1}-a_{12} \cdot c_{2}}{a_{11} \cdot a_{22}-a_{12} \cdot a_{21}}$

$M_{t}(Q, f) \equiv \frac{t}{T}=\frac{a_{11} \cdot c_{2}-a_{21} \cdot c_{1}}{a_{11} \cdot a_{22}-a_{12} \cdot a_{21}}$

where $M_{r}$ and $M_{t}$ are the modification factors introduced to represent the modification of the Fresnel reflection and transmission from the substrate by the presence of the sample layer and they contain all the information about the sample that could possibly be detected by the reflectivity experiment. $M_{r}$ and $M_{t}$ and the other parameters in Eqs.(10.a), (10.b), (11.a), (11.b), (12.a), (12.b) and their derivation are given in Appendix C.

The reflection and transmission coefficients $\mathbf{r}$ and $\mathbf{t}$ in Eqs.(11.a) and (11.b) are expected to give the correct relation between the reflectivity and the sample profile to the extent that the third and higher-order derivatives of the sample profile can be neglected. They are believed to be valid over the whole range of $Q$ values since they can reducn to the first-order result at large $Q$ values and they have accounted for the multiple reflections at small and medium $Q$ values as well. The reflectivity is obtained when the squared modulus is taken of Eq.(11.a), and obviously it is expressed as a functional of the profile without involving the phase of the reflection 
coefficient. This virtue might eliminate the need to know the phase at least for some classes of solutions, when a continuous profile is superimposed on a rigid substrate,

As the next step of study, the closed-form expressions for $\mathbf{r}$ and $\mathbf{t}$ will be evaluated both analytically and numerically for their accuracy and validity through the use of some model sample profiles. Once their goodness is confirmed, a computer scheme will be constructed to use them in the inverse calculation.

\section{ACKNOWLEDGEMENT}

This work was performed under the auspices of the U.S. Department of Energy, Division of Materials Sciences, Offices of Basic Energy Sciences, under contract W-31-109-ENG-38. 


\section{REFERENCES}

[1] G. P. Felcher, R. O. Hilleke, R. K. Crawford, J. Haumann, R. Kleb and G. Ostrowski, Rev. Sci. 58 (1987) 609.

[2] J. Penfold, R.C. Ward and W.G. Williams, J. Phys. E 20 (1987) 1411.

[3] J. Als-Nielsen, Nucl. Instrum. Methods 208 (1983) 545-548.

[4] R.M. Richardson and S.J. Roser, Liq. Cryst. 2 (1987) 797.

[5] K. Kjaer and J. Als-Nielsen, Thin Solid Films 159 (1988) 17-28.

[6] J. Jacobsson, in: Progress in Optics, E. Wolf, ed. (North Holland, Amsterdam, 1966).

[7] J. Lekner, Theory of Reflection, ( Martinus Nijhoff Publishers, 1987).

[ 8] G. P. Felcher, in: Proceedings of SPIE, Vol. 983, Thin Film Neutron Optical Devices, 16-17 August 1988, San Diego, California, USA.

[9] A. H. Compton and S. K. Allison, X-rays in Theory and Experiment, (D. Van Nostrand, 1935) pp 279-294.

[10] B. Farnoux, in: Proceedings of the Conference "Neutron Scattering in the Nineties", IAEA, Vienna (1985) p 205.

[11] M. L. Goldberger and F. Seitz, Phys. Rev. 71 (194'7) 294.

[12] C.-T. Tai, Dyadic Green's Functions..., (Intext Educational Publishers, 1971) pp 35-36.

[13] J. Als-Nielsen, Z. Phys. B 61 (1985) 411-414. 


\section{APPENDIX A DERIVATION OF THE OPEN-FORM SOLUTION FOR TEE WAVES}

The general solution to the second-order linear differential equation Eq.(1.b) is the sum of its particular solution and the general solution to the corresponding homogeneous equation. Denoting these by $U_{p}(z)$ and $U_{h}(z)$, respectively, one has the following equations for them

$$
\begin{aligned}
& U(z) \equiv U_{h}(z)+U_{p}(z) \\
& U_{h}^{\prime \prime}(z)+k_{o}^{2} U_{h}(z)=0, \text { for } z \leq 0 \\
& U_{h}^{\prime \prime}(z)+k_{s}^{2} U_{h}(z)=0, \text { for } 0 \leq z \\
& U_{p}^{\prime \prime}(z)+k_{o}^{2} U_{p}(z)=-\left(k^{2}-k_{o}^{2}\right) \mathrm{U}(z), \text { for } z \leq 0 \\
& U_{p}^{\prime \prime}(z)+k_{s}^{2} U_{p}(z)=0, \text { for } 0 \leq z
\end{aligned}
$$

The solution to Eqs.iA2.a) and (A2.b) under the continuity boundary conditions is the substrate reflection and transmission.

$$
\begin{aligned}
& U_{h}(z)=e^{i k_{o} z}+R \cdot e^{-i k_{o} z}, \text { for } \mathrm{z} \leq 0 \\
& U_{h}(z)=T \cdot e^{i k_{s} z}, \text { for } 0 \leq \mathrm{z}
\end{aligned}
$$

where $\mathrm{R}, \mathrm{T}$ are the Fresnel reflection and transmission coefficients for the substrate (Eqs.(4.d-e)).

The soluticn to Eqs.(A3.a) and (A3.b) can be obtained through the use of the half-space Green's functions $g_{1}\left(z, z^{\prime}\right)$ and $g_{2}\left(z, z^{\prime}\right)$ which satisfy the following equations 


$$
\begin{aligned}
& g_{1}^{\prime \prime}\left(z, z^{\prime}\right)+k_{o}^{2} g_{1}\left(z, z^{\prime}\right)=-\delta\left(z-z^{\prime}\right), \text { for } z \leq 0 \\
& g_{2}^{\prime \prime}\left(z, z^{\prime}\right)+k_{s}^{2} g_{2}\left(z, z^{\prime}\right)=0, \text { for } 0 \leq z \\
& \left.g_{1}\left(z, z^{\prime}\right)\right|_{z=0}=\left.g_{2}\left(z, z^{\prime}\right)\right|_{z=0} \\
& \left.g_{1}^{\prime}\left(z, z^{\prime}\right)\right|_{z=0}=\left.g_{2}^{\prime}\left(z, z^{\prime}\right)\right|_{z=0}
\end{aligned}
$$

Using standard procedure[12], namely, matching them at $z=z^{\prime}$, one finds

$$
\begin{aligned}
& g_{1}\left(z, z^{\prime}\right)=\frac{i}{2 k_{o}}\left[e^{i k_{o}\left|z^{\prime}-z\right|}+R \cdot e^{-i k_{o}\left(z^{\prime}+z\right)}\right], \text { for } z \leq 0 \\
& \mathrm{~g}_{2}\left(z, z^{\prime}\right)=\frac{i}{2 k_{o}} T \cdot e^{i k_{s} z-i k_{o} z^{\prime}}, \text { for } 0 \leq \mathrm{z}
\end{aligned}
$$

With these Green's functions, the particular solution is equal to the integration of the product of the source term (i.e. the sample profile $\mathrm{k}^{2} \cdot \mathrm{k}_{0}{ }^{2}$ ) with the Green's functions. The interration limits are from - $d$ to 0 , namely, over the sample region. Noting that the RHS of Eq.(A5.a) contains a minus sign, one has

$$
\begin{aligned}
& U_{p}(z)=\int_{-d}^{0} d z^{\prime} \cdot\left(k^{2}-k_{o}^{2}\right) U\left(z^{\prime}\right) g_{1}\left(z, z^{\prime}\right), \text { for } z \leq 0 \\
& U_{p}(z)=\int_{-d}^{0} d z^{\prime} \cdot\left(k^{2}-k_{o}^{2}\right) U\left(z^{\prime}\right) g_{2}\left(z, z^{\prime}\right), \text { for } 0 \leq \mathrm{z}
\end{aligned}
$$

Substituting $U_{h}$ from Eqs.(A4) and Up from Eqs.(A7) into Eq.(A1), one obtains the open-form solution for the wave function $U(z)$ 
APPENDIX B. DERIVATION OF THE CLOSED-FORM SOLUTION FOR THE

MULTIPLE-REFLECTION FUNC'TIONS A(Z) AND B(Z)

The functions $a(z)$ and $b(z)$ in Eqs.(7.b) and (7.c) satisfy differential equations

$$
\begin{aligned}
& a^{\prime \prime}(z)-C_{a} a^{\prime}(z)+f(z) a(z)=0 \\
& b^{\prime \prime}(z)-C_{b} b^{\prime}(z)+f(z) b(z)=0 \\
& C_{a}=\left[\ln \left(f e^{-i 2 k_{o} z}\right)\right]^{\prime} \\
& C_{b}=\left[\ln \left(f e^{i 2 k_{o} z}\right)\right]^{\prime}
\end{aligned}
$$

Substituting Eqs.(7.b) and (7,c) into Eqs.(B1.a) and (B1.b), one gets

$$
\begin{aligned}
& A^{\prime \prime}(z)-C_{a} A^{\prime}(z)+f(z) A(z)=-\frac{i}{2 k_{o}} f(z) \int_{-d}^{z} d z^{\prime} \cdot f\left(z^{\prime}\right)\left(1+r e^{-i 2 k_{o} z^{\prime}}\right) \\
& B^{\prime \prime}(z)-C_{b} B^{\prime}(z)+f(z) B(z)=-\frac{t}{T} \frac{i}{2 k_{o}} f(z) \int_{z}^{0} d z^{\prime} \cdot f\left(z^{\prime}\right)\left(e^{i 2 k_{o} z^{\prime}}+R\right)
\end{aligned}
$$

First let

$$
\begin{aligned}
& A^{\prime}(z) \equiv f(z) \cdot \int_{-d}^{z} d z^{\prime} \cdot \frac{E\left(z^{\prime}\right)}{f^{2}\left(z^{\prime}\right)} \\
& B^{\prime}(z) \equiv-f(z) \cdot \int_{z}^{0} d z^{\prime} \cdot \frac{F\left(z^{\prime}\right)}{f^{2}\left(z^{\prime}\right)}
\end{aligned}
$$


Substituting Eqs.(B3.a) and (B3.b) into Eqs.(B2.a) and (B2.b), one has

$$
\begin{aligned}
E^{\prime}(z)+\left(2 i k_{o}-2 \frac{f^{\prime}}{f}\right) E(z)= & -f^{3} \cdot \int_{-d}^{z} d z^{\prime} \frac{E\left(z^{\prime}\right)}{f^{2}\left(z^{\prime}\right)}- \\
& \frac{i}{2 k_{o}} f^{3}\left(1+r e^{-i 2 k_{o} z}\right) \\
F^{\prime}(z)+\left(-2 i k_{o}-2 \frac{f^{\prime}}{f}\right) F(z)= & f^{3} \cdot \int_{z}^{0} d z^{\prime} \frac{F\left(z^{\prime}\right)}{f^{2}\left(z^{\prime}\right)}+ \\
& \frac{t}{T} \frac{i}{2 k_{o}} f^{3}\left(e^{i 2 k_{o} z}+R\right)
\end{aligned}
$$

Then, one defines $G(z)$ and $H(z)$ as

$$
\begin{aligned}
& E(z) \equiv f^{2} G(z) e^{-i 2 k_{o} z} \\
& F(z) \equiv f^{2} H(z) e^{i 2 k_{o} z}
\end{aligned}
$$

and substitutes Eqs.(B5.a) and (B5.b) into Eqs.(B4.a) and (B4.b) to fir.d

$$
\begin{aligned}
& G^{\prime}(z)=-f e^{i 2 k_{o} z} \int_{-d}^{z} d z^{\prime} \cdot G\left(z^{\prime}\right) e^{-i 2 k_{o} z^{\prime}}-\frac{i}{2 k_{o}} f \cdot\left(e^{i 2 k_{c} z}+r\right) \\
& H^{\prime}(z)=f e^{-i 2 k_{o} z} \int_{z}^{0} d z^{\prime} \cdot H\left(z^{\prime}\right) e^{i 2 k_{o} z^{\prime}}+\frac{t}{T} \frac{i}{2 k_{o}} f \cdot\left(1+\mathrm{Re}^{-i 2 k_{o} z}\right)
\end{aligned}
$$

The variation of Eqs.(B6.a) and (B6.b) is due to two contributions: one is the variation of $e^{ \pm i 2 k_{0} z}$, and the other is the variation of $\mathrm{f}(\mathrm{z})$ as a function of $\mathrm{z}$. The first part can not be approximated while the second part can when the third and higher derivatives of $f(z)$ are neglected. Naturilly, one would try 
to separate them from each other so that approximations could be made. To do so, one writes

$$
\begin{aligned}
& \int_{-d}^{2} d z^{\prime} \cdot G\left(z^{\prime}\right) e^{-i 2 k_{o} z^{\prime}} \equiv f(z) \cdot\left[g(z)+l(z) e^{-i 2 k_{o} z}\right] \\
& \int_{z}^{0} d z^{\prime} \cdot H\left(z^{\prime}\right) e^{i 2 k_{o} z^{\prime}} \equiv f(z) \cdot\left[h(z)+m(z) e^{i 2 k_{o} z}\right]
\end{aligned}
$$

Substituting Eqs.(B7.a) and (B7.b) into Eqs.(B6.a) and (B6.b) and equating the corresponding coefficients yield four differential equations

$$
\begin{aligned}
& g^{\prime \prime}(z)+2 i k_{o} g^{\prime}(z)+f(z) g(z)=-\frac{i}{2 k_{o}} f(z) \\
& l^{\prime \prime}(z)-2 i k_{o} l^{\prime}(z)+f(z) l(z)=-r \cdot \frac{i}{2 k_{o}} f(z) \\
& h^{\prime \prime}(z)-2 i k_{o} h^{\prime}(z)+f(z) h(z)=-\frac{t}{T} R \cdot \frac{i}{2 k_{o}} f(z) \\
& m^{\prime \prime}(z)+2 i k_{o} m^{\prime}(z)+f(z) m(z)=-\frac{t}{T} \cdot \frac{i}{2 k_{o}} f(z)
\end{aligned}
$$

Eqs.(B8.a) to (B8.d) can be cast in a single form

$$
\begin{aligned}
& P^{\prime \prime}(z)+\alpha P^{\prime}(z)+f(z) P(z)=\beta \cdot f(z) \\
& \alpha=2 i k_{o} \text { and } \beta=1 \text { for } P(z)=g(z) \\
& \alpha=-2 i k_{o} \text { and } \beta=\mathrm{r} \text { for } P(z)=l(z) \\
& \alpha=-2 i k_{o} \text { and } \beta=(t / T) R \text { for } P(z)=h(z) \\
& \alpha=2 i k_{o} \text { and } \beta=t / T \text { for } P(z)=m(z)
\end{aligned}
$$


This is the final set of equations which we want to solve approximately. The third and higher derivatives of $f(z)$ will be ignored, namely, $f(n)=0$ for $n>2$. In addition, one can write

$$
P^{\prime}(z)=f(z) \cdot S[f(z)]
$$

which can be easily proven. Therefore, to the same order of approximation as is the negligence of the third and higher derivatives of $f(z)$, one can ignore $\mathrm{S}^{(\mathrm{n})}$ for $\mathrm{n}>3$ since $\left.\mathrm{S}^{\prime}[\mathrm{f}(\mathrm{z})] \sim \mathrm{f}^{\prime} \mathrm{z}\right)$ according to Eq.(B9.a). Differentiating Eq.(B10) four times and using the above approximations, one obtains

$$
P^{(5)}=6 f^{\prime \prime}(z) \cdot S^{\prime \prime}+4 f^{\prime}(z) \cdot S^{\prime \prime \prime}
$$

Using Eq.(B10) to eliminate S from Eq.(B11), one gets

$$
\begin{aligned}
& P^{(5)}=4 \rho P^{(4)}+6\left(\rho^{\prime}-\rho^{2}\right) P^{\prime \prime \prime}-24 \rho^{\prime} \rho P^{\prime \prime}+\left(2 \rho^{4}-4 \rho \rho^{\prime \prime}-6 \rho^{2}+12 \rho \rho^{\prime 2}\right) P^{\prime} \\
& \rho=\rho(z) \equiv(\ln f)^{\prime}
\end{aligned}
$$

Then, we differentiate Eq.(B9.a) once, twice, and three times to obtain three equations containing the third, the fourth and the fifth derivatives, respectively. Substiluting Eq.(B12.a) into the equation just derived which contains the fifth derivative, one can solve for the fourth derivative in terms of the third, the second and the first derivatives. In turn, by substituting this expression into the equation derived above which contains the fourth derivative of $P$, one ends up with an expression for the third derivative of $P$ in terms of the second and the first derivatives of $P$. Keeping going one more step, one arrives at a formula for the second derivative of $\mathrm{P}$ in terms of the first derivative of $\mathrm{P}$. Finally, one substitutes this expression for $\mathrm{P}^{\text {" into }}$ Eq.(B9.a) to get a first-order differential equation in the standard form 


$$
\begin{aligned}
P^{\prime}(z)=[ & P(z)-\beta] f(z) \cdot \Omega(\alpha, z) \\
\Omega(\alpha, z) \equiv & \left\{\alpha^{3}+3 \alpha^{2} \rho+3 \alpha \rho^{2}-3 \rho^{3}-5 \alpha \rho^{\prime}-17 \rho \rho^{\prime}-\rho^{\prime \prime}-2 \alpha f\right\}+ \\
& \left\{-\alpha^{4}-4 \alpha^{3} \rho-6 \alpha^{2} \rho^{2}+2 \rho^{4}+6 \alpha^{2} \rho^{\prime}+24 \alpha \rho \rho^{\prime}+\alpha \rho^{\prime \prime}\right. \\
& \left.-6 \rho^{\prime 2}+12 \rho^{\prime} \rho^{2}+3 \alpha^{2} f+3 \alpha \rho \rho^{f}+f \rho^{2}-f^{2}-3 f \rho^{\prime}\right\}
\end{aligned}
$$

Letting

$$
\Delta(z) \equiv P(z)-\beta
$$

then one has

$$
\begin{aligned}
& \frac{\Delta^{\prime}(z)}{\Delta(z)}=f(z) \Omega(\alpha, z) \\
& \Delta(-d)=-\beta \text { for } \mathrm{g}(\mathrm{z}) \text { and } \mathrm{l}(\mathrm{z}) \\
& \Delta(0)=-\beta \text { for } \mathrm{h}(\mathrm{z}) \text { and } \mathrm{m}(\mathrm{z})
\end{aligned}
$$

The solution of Eq.(B15.a) follows easily 


$$
\begin{aligned}
& g(z)=\frac{i}{2 k_{o}}\left[\begin{array}{ll}
\int^{z} d z^{\prime} \cdot f\left(z^{\prime}\right) \Omega\left(2 i k_{o}, z^{\prime}\right) & \\
e^{-d} & -1
\end{array}\right] \\
& l(z)=\frac{i}{2 k_{o}} r\left[e^{\int^{-d} d z^{\prime} \cdot f\left(z^{\prime}\right) \Omega\left(-2 i k_{o}, z^{\prime}\right)}-1\right] \\
& h(z)=\frac{i}{2 k_{o}} \frac{t}{T} R\left[e^{-\int^{0} d z^{\prime} \cdot f\left(z^{\prime}\right) \Omega\left(-2 i k_{o}, z^{\prime}\right)}-1\right] \\
& m(z)=\frac{i}{2 k_{o}} \frac{t}{T}\left[e^{-\int^{0} d z^{\prime} \cdot f\left(z^{\prime}\right) \Omega\left(2 i k_{o}, z^{\prime}\right)}-1\right]
\end{aligned}
$$

and substituting Eqs.(B16.a) to (B16.d) into Eqs.(B3.a) and (B3.b) and integrating them, one gets the result in Eqs.(8.a) and (8.b).

\section{APPENDIX C. DERIVATION OF THE REFLECTION AND TRANSMISSION COEFFICIENTS $\mathbf{r}$ AND $t$}

From Eqs.(6.a) and (6.b), one sees that once the wave function $U(z)$ inside the sample is obtained in a closed form, $r$ and $t$ can be put in a closed form directly. Substituting Eqs.(8.a) and (8.b) into Eqs.(7.b) and (7.c), one has 


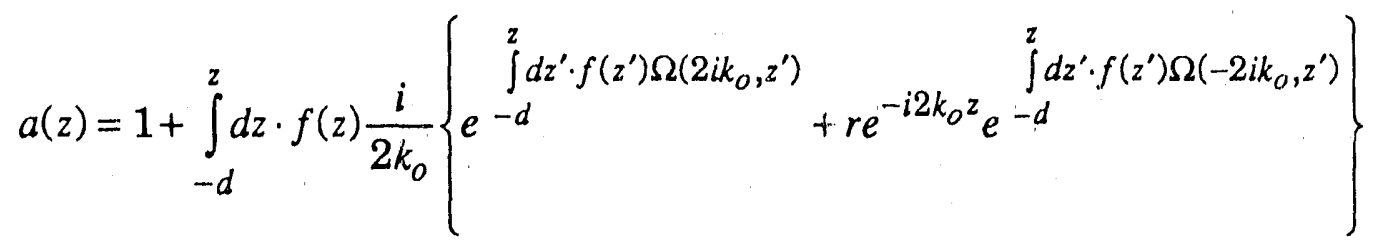

$$
\begin{aligned}
& b(z)=\frac{t}{T} R+\int_{z}^{0} d z \cdot f(z) \frac{i}{2 k_{o}} \frac{t}{T}\left\{\mathrm{R} e^{-\int^{0} d z^{\prime} \cdot f\left(z^{\prime}\right) \Omega\left(-2 i k_{o}, z^{\prime}\right)}+e^{i 2 k_{o} z} e^{-\int^{0} d z^{\prime} \cdot f\left(z^{\prime}\right) \Omega\left(2 i k_{o}, z^{\prime}\right)}\right\}
\end{aligned}
$$

Substituting Eq.(7.a) into Eqs.(6.a) and (6.b), one has

$$
\begin{aligned}
& r-R=\frac{i}{2 k_{o}} \int_{-d}^{0} d z^{\prime} \cdot f\left(z^{\prime}\right) \cdot\left[a\left(z^{\prime}\right)+b\left(z^{\prime}\right) e^{-i 2 k_{o} z^{\prime}}\right]\left(e^{i 2 k_{o} z^{\prime}}+R\right) \\
& t-T=\frac{i}{2 k_{o}} T \cdot \int_{-d}^{0} d z^{\prime} \cdot f\left(z^{\prime}\right) \cdot\left[a\left(z^{\prime}\right)+b\left(z^{\prime}\right) e^{-i 2 k_{o} z^{\prime}}\right]
\end{aligned}
$$

Substituting Eqs.(C1.a) and (C1.b) into Eqs.(C2.a) and (C2.b), one obtains a set of simultaneous equations for $r$ and $t$

$$
\begin{aligned}
& a_{11} \frac{r}{R}+a_{12} \frac{t}{T}=c_{1} \\
& a_{21} \frac{r}{R}+a_{22} \frac{t}{T}=c_{2}
\end{aligned}
$$

where, with $Q=2 k_{0}$, the coefficients are 


$$
\begin{aligned}
& a_{11}=1+\frac{1}{Q^{2}} \int_{-d}^{0} d z^{\prime} f\left(z^{\prime}\right)\left(e^{i Q z^{\prime}}+R\right) \tau\left(z^{\prime}\right) \\
& a_{22}=1-\frac{i}{Q} R \int_{-d}^{0} d z^{\prime} f\left(z^{\prime}\right) e^{-i Q z^{\prime}}+\frac{1}{Q^{2}} \int_{-d}^{0} d z^{\prime} f\left(z^{\prime}\right) e^{-i Q z^{\prime}} \zeta\left(z^{\prime}\right) \\
& a_{12}=-\frac{i}{Q} \int_{-d}^{0} d z^{\prime} f\left(z^{\prime}\right)\left(1+\operatorname{Re}^{-i Q z^{\prime}}\right)+\frac{1}{Q^{2}} \int_{-d}^{0} d z^{\prime} \frac{f\left(z^{\prime}\right)}{R}\left(1+R e^{-i Q z^{\prime}}\right) \zeta\left(z^{\prime}\right) \\
& a_{21}=\frac{R}{Q^{2}} \int_{-d}^{0} d z^{\prime} f\left(z^{\prime}\right) \tau\left(z^{\prime}\right) \\
& c_{1}=1+\frac{i}{Q} \int_{-d}^{0} d z^{\prime} \frac{f\left(z^{\prime}\right)}{R}\left(e^{i Q z^{\prime}}+R\right) \chi\left(z^{\prime}\right) \\
& c_{2}=1+\frac{i}{Q} \int_{-d}^{0} d z^{\prime} f\left(z^{\prime}\right) \chi\left(z^{\prime}\right) \\
& \chi(z)=1+\frac{i}{Q} \int_{-d}^{z} d z \cdot f(z) e^{-d} \int^{-d} d z^{\prime} \cdot f\left(z^{\prime}\right) \Omega\left(i Q, z^{\prime}\right)
\end{aligned}
$$

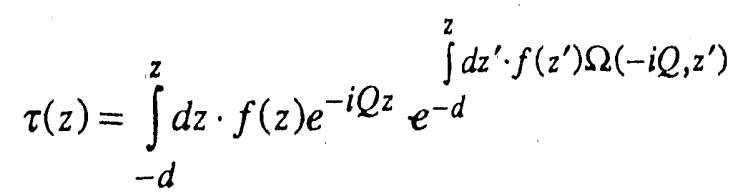

$$
\begin{aligned}
& \zeta(z)=\int_{z}^{0} d z \cdot f(z)\left\{R e^{-\int^{-\int} d z^{\prime} \cdot f\left(z^{\prime}\right) \Omega\left(-i Q, z^{\prime}\right)}+e^{i Q z^{z}} e^{-\int^{0} d z^{\prime} \cdot f\left(z^{\prime}\right) \Omega\left(i Q, z^{\prime}\right)}\right\}
\end{aligned}
$$


Directly solving Eqs.(C3.a) and (C3.b) gives

$$
\begin{aligned}
& r=M_{r}(Q, f) \cdot R \\
& t=M_{t}(Q, f) \cdot T
\end{aligned}
$$

with

$$
\begin{aligned}
& M_{r}(Q, f) \equiv \frac{r}{R}=\frac{a_{22} \cdot c_{1}-a_{12} \cdot c_{2}}{a_{11} \cdot a_{22}-a_{12} \cdot a_{21}} \\
& M_{t}(Q, f) \equiv \frac{t}{T}=\frac{a_{11} \cdot c_{2}-a_{21} \cdot c_{1}}{a_{11} \cdot a_{22}-a_{12} \cdot a_{21}}
\end{aligned}
$$

where $M_{r}$ and $M_{t}$ are introduced to represent the modification of the Fresnel's reflection and transmission from the substrate by the presence of the sample layer and they contain all the information about the sample that would by any chance be detected by the reflectivity experiment. 


\section{IIST OF FIGURES}

Figure 1. The geometry of $x$-ray and neutron reflection and transmission, where $1, \mathbf{r}$ and $\mathbf{t}$ denote the amplitudes of the incident, reflected and transmitted waves, respectively, $\mathbf{d}$ is the sample thickness, $\theta$ the incident angle, ko, $\mathbf{k}$ and $\mathbf{k s}$ are the wave numbers in the $\mathbf{z}$-direction in the free space, the sample layer and the substrate, respectively, and the arrows represent the directions of waves. 
Figure 1:

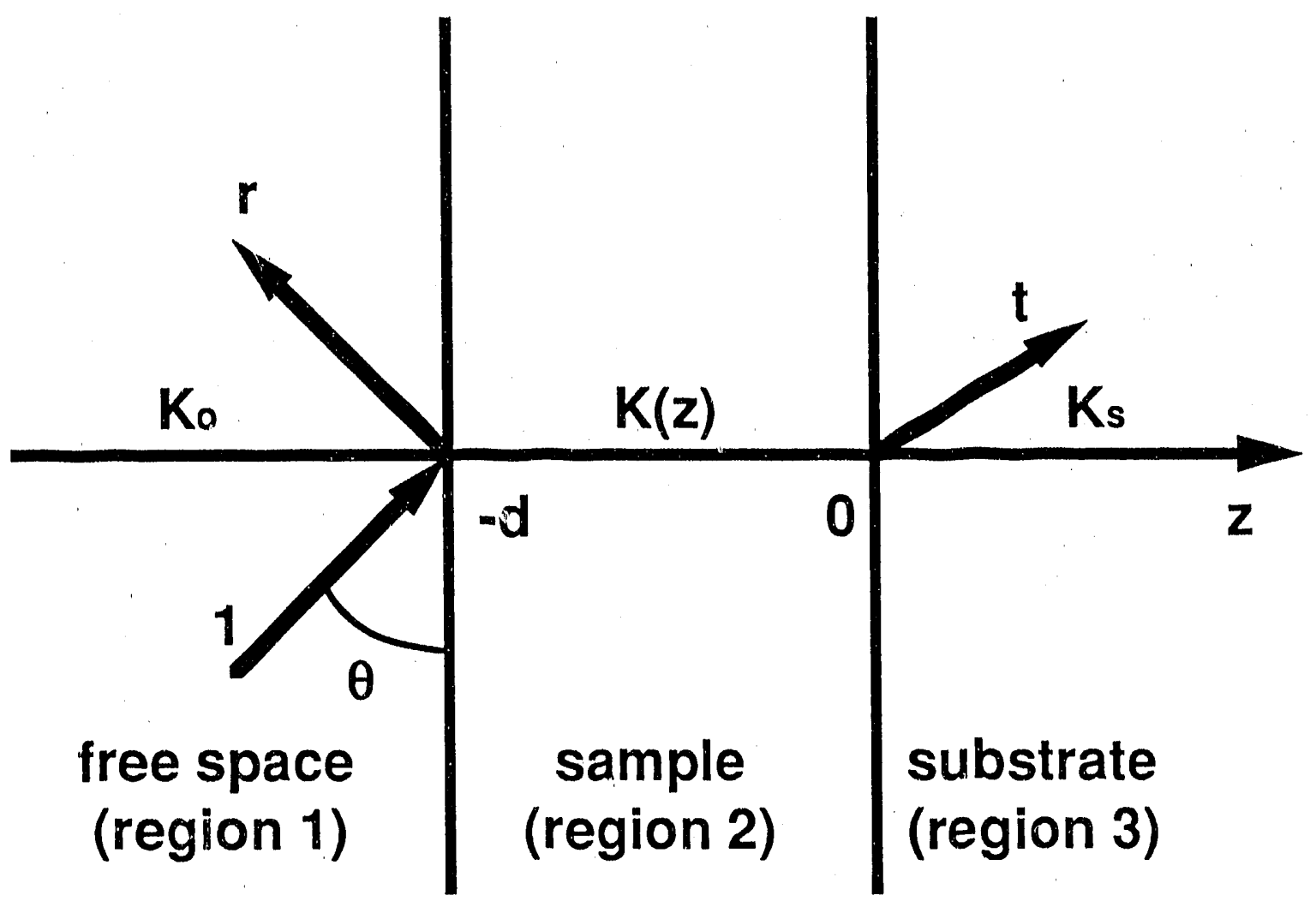



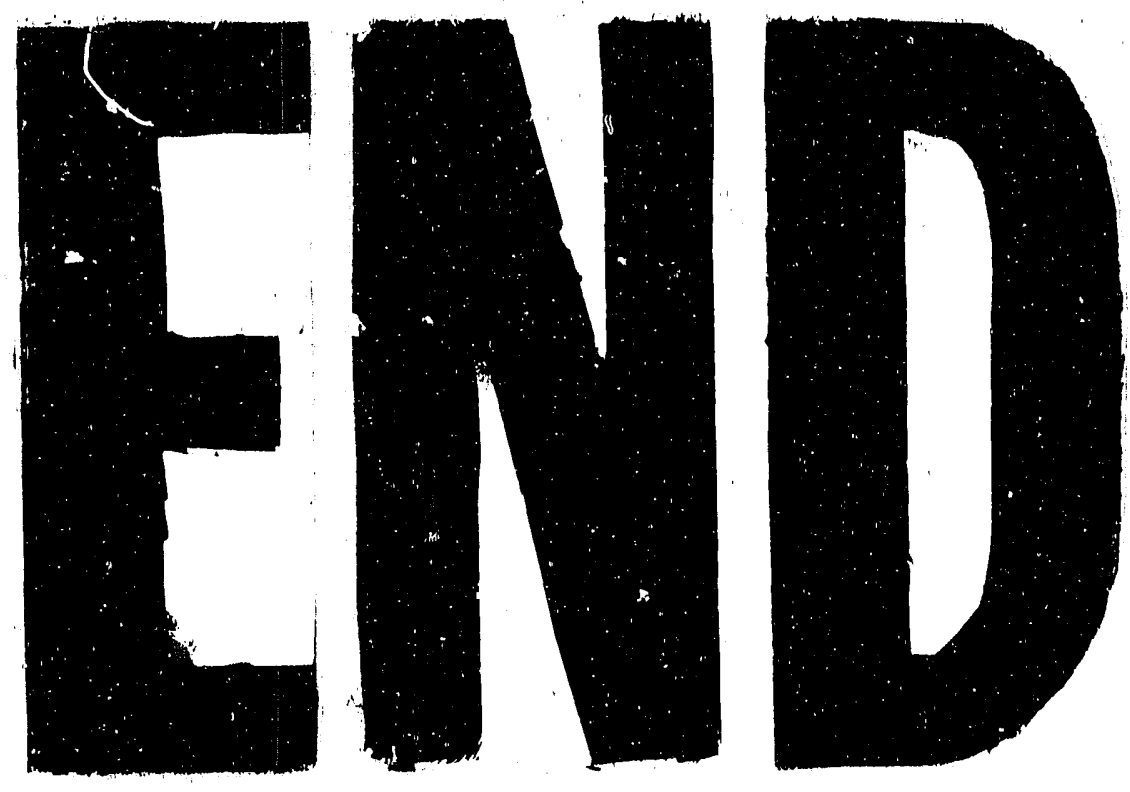

4
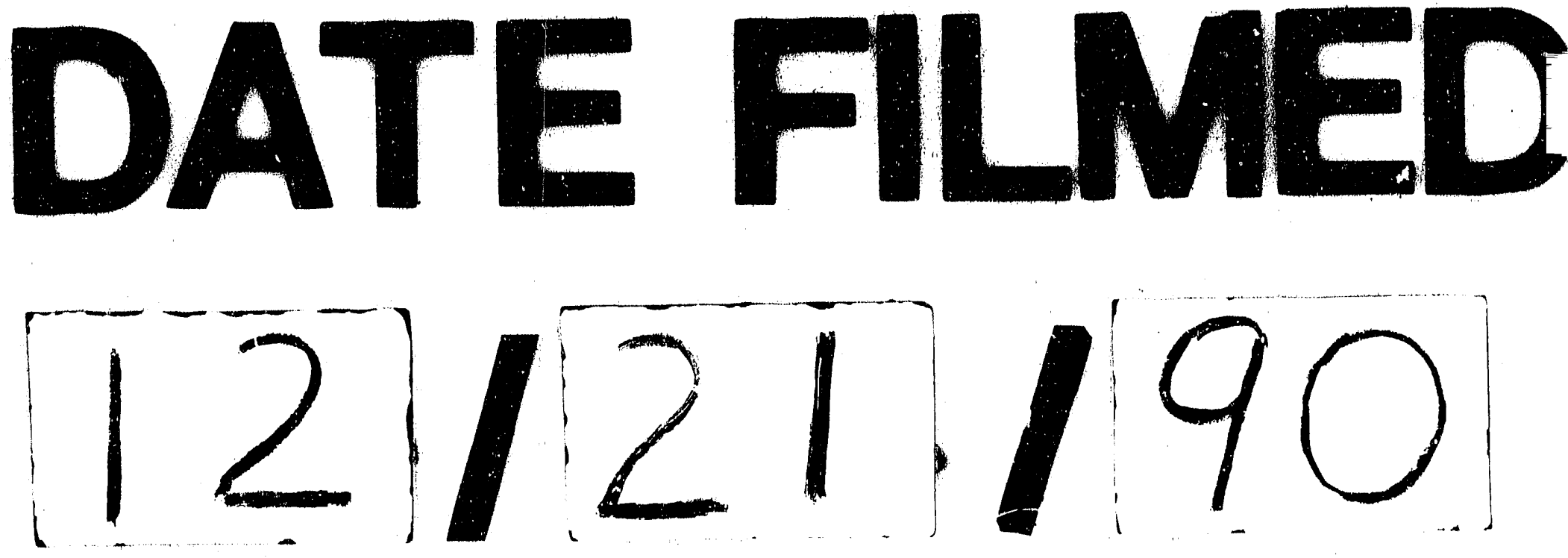